\title{
FEMMINISMO E BRIGANTAGGIO: LO SGUARDO DIFFERENTE DI MARIA ROSA CUTRUFELLI
}

\author{
FEMINISM AND BANDITRY: ROSA CUTRUFELL'S DIFFERENT EYE
}

Silvio Cosco

Universidad de Roma

\section{RIASSUNTO:}

La letteratura sul brigantaggio è in debitocon La Briganta (1990) di M. Rosa Cutrufelli. Il romanzo, sintesi della teoría storicofeminista della scrittrice, racconta la storiadi una brigantessa e rende omaggio a tuttele donne che hanno lottato contro alla logica patriarcale e, in particular modo, a queste coraggiose guerrigliere del XIX secolo, che la storia troppo spesso cataloga come "drude dei briganti".

\section{PAROLE CHIAVE:}

Brigantesse, femminismo, donne, patriarcato, Cutrufelli, letteratura.

\section{ABSTRACT:}

The literature about South Italy's "brigantesse" is indebted to La Briganta (1990) by Maria Rosa Cutrufelli. The novel, synthesis of the historical and feminist theories of Cutrufelli, telling the story of a woman brigand, pays tribute to all women who have fought against the logic of patriarchy and especially to these brave women guerrillas too often catalogued as "concubines of brigands".

\section{KEY WORD:}

Brigands, femminism, patriarchy, Cutrufelli, literature, ri-historied. 
La letteratura sul brigantaggio ha visto ampliarsi, soprattutto negli ultimi anni con il sorgere di una serie testi di ri-storicizzazione sull'unificazione italiana, lo spazio nel settore editoriale. Si è tornati a narrare le gesta dei briganti, e finalmente, delle brigantesse: donne che in passato si cercò di catalogare come "drude dei briganti" "concubine" o "brigantesse per amore", archiviando così la loro partecipazione coscienziosa alla lotta di resistenza delle popolazioni meridionali al nuovo potere piemontese. La rilettura data dalla nuova letteratura sulle brigantesse è senz'altro debitrice alla pubblicazione de La Briganta di Maria Rosa Cutrufelli, datata 1990 e tutt'ora ineguagliabile perla del genere. La Cutrufelli, scrittrice e giornalista messinese di successo (i suoi libri sono tradotti in 20 lingue), si cimenta nella narrazione di un personaggio fittizio ma realistico, colmando il vuoto del silenzio sul brigantaggio femminile. Lei, donna attiva nel movimento femminista e attenta ai problemi della condizione femminile, indagati in fondamentali testi degli anni 70-80, ma anche dotata di un personale punto di vista storico esposto in L'Unità d'Italia guerra contadina e nascita del sottosviluppo del Sud (1974), riesce a riunire questi interessi nel suo primo romanzo.

La Cutrufelli è cosciente che il brigantaggio fu una reazione popolare e contadina ad un' unificazione vissuta dal sud come un' annessione al Piemonte da cui il meridione non solo non trasse alcun vantaggio ma subì indelebili danni socio-economici. Inoltre, la scrittrice siciliana sostiene che il periodo del brigantaggio postunitario essendo politicamente autonomo, permise alle donne di ricoprire un ruolo di straordinaria importanza, ergendosi spesso a capo di bande. «Non sono al seguito degli uomini delle bande, ma fanno parte dei quadri organizzati del brigantaggio», scrive nel suo saggio (Cutrufelli, 1974). Queste donne si ritagliarono, all'interno di un secolo in cui si confinava la donna a "angelo del focolare", uno spazio di libertà mai visto con atteggiamenti che erano «chiari insulti alla morale dell'epoca: vestite spesso col più pratico abbigliamento maschile, le gambe divaricate (...) fucili ed armi tenute con la massima disinvoltura» (Cutrufelli, cit.). Non è casuale, come fanno notare anche Di Giulio (2005) e Rossi (1993) che la prima "personaggia" della scrittrice siciliana sia una brigantessa: la scrittrice viene indotta a questa scelta dopo un'accurata opera di documentazione presso l'Archivio di Stato. Tra le carte processuali dell'epoca, l'autrice scoprì i nomi di donne straordinarie che scelsero la lotta armata in funzione antiunitaria e vennero sepolte dall'oblio e dalla mistificazione: le brigantesse, inferiori agli uomini solo numericamente. $\mathrm{Ne}$ brigantaggio post-unitario molte donne si diedero alla macchia e furono attive nelle bande partecipando alle azioni militari e spesso dirigendole, senza che nessun uomo ne mettesse in discussione il ruolo. Eppure la storia, scritta e narrata dagli uomini e dai vincitori, ha distorto l'essenza del brigantaggio femminile, a volta dileggiandoloe volte mitizzandolo, dandole una connotazione eterica. Così, non deve sorprendere che sia un "realistico" romanzo del 1990 a raccontare il coraggio e la dignità di queste donne attraverso la fittizia autobiografia di Margherita, una brigantessa anomala ma credibile. Infatti, "la Briganta” non è una donna del popolo, né un'operaia né una contadina, ma proviene da una famiglia aristocratica. Margherita è la protagonista di una breve epopea da fuorilegge che subentra ad un'infanzia da nobile, ad una vita da altri pianificata. Diviene brigantessa dopo essersi liberata di un matrimonio coercitivo: il gesto estremo dell'omicidio del marito la porta a darsi alla macchia. Tutto si svolge sullo sfondo della Sicilia postunitaria lacerata dall'incuria istituzionale del nuovo stato. Nel 1883, dopo vent'anni di carcere, la Briganta matura la decisione di affidarsi alla stesura delle sue memorie per liberare la sua interiorità repressa. La scelta della autobiografia come espediente letterario per il racconto è permesso dal rango e dall?istruzione che ha ricevuto la protagonista, e che si differenzia dalla sue compagne d'armi. La scelta della Cutrufelli di raccontare un brigantessa di "sangue blu", comunque, non appare forzata alla luce di una presenza seppur minoritariama documentata di donne istruite fra le brigantesse. Nonostante l'operazione della protagonista-narratrice sia riattraversare la propria vita scrivendone un memoriale, "la scrittura" non è l'elemento caratterizzante di questa donna, che avrebbe benissimo potuto essere un'analfabeta. Quella compiuta dalla Cutrufelli/Briganta è piuttosto una scelta narrativa che ci appare obbligata e rispondente alla necessita di "fare storia". È necessario che sia una donna a raccontare, seppur in modo romanzato, ciò che firme maschili nascosero: il coraggio di queste donne 800esche di lottare non solo per motivi politici o contingenti alla loro situazione ma per un vero e proprio affrancamento da imposizioni sociali e culturali.

«Non sono una donna di briganti, ma una briganta» (Cutrufelli, 19901) dice "La Bizzarra", una delle donne militanti della banda di Margherita. In questa frase la scrittrice rinchiude la necessità per queste donne di rivendicare la propria identità di fronte alla storia e alle leggi, sempre obbedienti alle logiche del patriarcato. Così la Margherita di Cutrufelli parla, o meglio, scrive per tutte. D'altronde, la stessa scrittrice messinese sostiene che «la scrittura (...) è l'arma migliore per la costruzione di un'identità» (Cutrufelli, 2005). La Briganta come spesso succede nei romanzi della Cutrufelli, ci racconta la Storia con la esse maiuscola attraverso la sua vicenda personale. E lo fa con lo "sguardo differente" della donna: di chi «anche nella propria terra, anche nella propria casa, può sentirsi in esilio, straniera e nemica, sperimentando in questo modo direttamente - e a volte duramente - la necessità del cambiamento, di una frattura culturale, di un dialogo con le altre» (Cutrufelli, 2002). La "sguardo differente" permette a chiunque sia capace di averlo, di smascherare, condannando, quelle che la scrittrice siciliane definisce le «molestie storiche» (Cutrufelli, 2005), sulla linea della più ampia categoria di «molestie testuali» di Elaine Showalter (1986): si tratta tutta quell'opera di storicizzazione misogina che ha colpito le donne ogni volta che, nel

1 A partire da qui, tutte le citazioni non diversarmente specificate saranno estratte dalla novella La Briganta. 
corso della storia, hanno provato a sovvertire le gerarchie imposte patriarcato. Con un espediente letterario la scrittrice e femminista Cutrufelli permette ad una brigantessa di raccontare tutte le brigantesse: questo risponde alle necessità di poter finalmente prendere la parola e disporre, come sosteneva Virginia Woolf di «Una stanza tutta per sé».

Margherita inizia il suo racconto da «sepolta viva» nel carcere, quasi rimpiangendo una morte eroica in battaglia. Lo scrivere, per lei, assomiglia ad un grido di libertà: «che la mia voce esca dalla cella che trattiene il mio corpo. Oggi quest'evasione mi è permessa: ho carta, inchiostro, penna e un passato da narrare e recuperare dal fondo del mio stesso oblio» (Cutrufelli, 1990). «Forse domani qualcuno capirà (...) che enorme libertà sia prendere la parola», sospira Margherita confidando nella volontà di conoscenza dei posteri. In carcere, la nostra Briganta ha subito oltre alla reclusione lo studio violento e razzista dell'antropologia lombrosiana volta a codificare la fisiognomia criminale e a identificarla in certi tratti meridionali. La scrittura delle proprie memorie appare per Margherita una rivincita pericolosa, una «cosa ardimentosa, forse ancora più che l'andar briganteggiando per i monti». Questo proprio perché è cosciente delle "molestie testuali" che lei come altre donne dovrà subire per gli spazi di libertà conquistati sia con le armi e che con la penna: «il coraggio è una virtù superflua in una donna - scrive - una virtù strappata all'uomo che in noi, forse necessariamente, diventa vizio e disperazione».

La protagonista del romanzo cresce in un non precisato paese del Regno delle due Sicilie, dove vive tranquillamente fino ai 22 anni «ignara di odi e ingiustizie». La madre, napoletana, la cresce con difficoltà in un paesino lontano dalla capitale, dove in passato aveva tenuto un salone, un luogo di incontri di intellettuali e rivoluzionari del 1799. Il padre «era diverso - racconta Margherita - a lui importava soltanto l'ascendenza aristocratica della donna che aveva sposato, e per questo tollerava anche le sue stravaganze. Prima fra tutte, l'avermi dato un'istruzione». In questo contesto è ovvio che la morte della madre lasci Margherita in una condizione di smarrimento. Il padre si libera, letteralmente, dei due figli: il fratello Cosimo viene mandato a studiare a Napoli, lei viene "ceduta" in matrimonio. Margherita è costretta ad accettare: «mia madre mi aveva insegnato a leggere ed a sognare ma non a difendermi - lamenta - E mio marito cominciò a disfare ciò che mia madre aveva costruito».

Il "disfacimento" dell'emancipazione della protagonista è addirittura materializzato dai libri fatti sparire dal marito («Né più libri né musica c'erano nella mia nuova vita matrimoniale»). Questa condizione di umiliazione quotidiana, di segregazione domestica, spinge la protagonista ad un impulso di liberazione che appare inevitabile: una notte uccide il marito nel sonno, colpendolo con un mattone. Così la vita "fuorilegge" di Margherita inizia «con un marchio di sangue». Lascia il palazzo prima che la serva Filomena rinvenga il cadavere e scappa correndo nei boschi. Al risveglio nella "macchia" la testa «finalmente sgombra, libera» contrasta con il pensiero di «quell'aria chiusa, stagnante della camera da letto» che gli sottraeva «perfino lo spazio dei sogni». Nel bosco, sola, ripensa alle passeggiate con il fratello Cosimo, ora «soldato di re Francesco, come si diceva allora. O brigante, come si disse poi». Siamo nel 1861, ed ancora vivo il ricordo della spedizione dei mille condotta agevolmente da Garibaldi, «il Dittatore»: «i liberali gli aprivano le vie mentre il popolo -scrive la Briganta - veniva sedotto con le promesse più inverosimili: esenzione della leva, esenzione delletasse». Margherita narra il disincanto di tanti meridionali, esemplificati dal fratello Cosimo. Anche lui aveva aderito e creduto alla spedizione garibaldina per essere poi,insieme ai suoi compagni, abbandonato al proprio destino: «nessuna prospettiva - scrive Margherita - Avevano fatto domanda per entrare nell'esercito regolare, ma le domande venivano respinte quasi tutte». Così che di Garibaldi, il "traditore", vengono bruciate le effigie in piazza. Con questa quadro socio-politico nello sfondo, Margherita si è convertita in una assassina. Eppure non mostra nessun segnale di cedimento né pentimento ma anzi, si vede rassicurata da questo non poter tornare più indietro. In fondo, la sua vita matrimoniale era la peggiore delle carceri. Nella "macchia" rincontra l'amato fratello, ora brigante nella banda di Carmine Spaziante. Nonostante l'immenso affetto che lega i due, Cosimo, figlio del suo tempo, si mostra incapace di capire le esigenze della sorella quanto le racconta l'accaduto.

«Ti porterò via subito - le dice - Il convento di Longapietra, tu lo conosci...La madre superiora è donna di grande intelligenza e carità. Con lei decideremo il da farsi, e finché non avremmo deciso là dentro sarai al sicuro». Margherita, delusa da parole che mai si sarebbe aspettata dal fratello, deve constatare come la sua necessità di libertà si scontri con l'istinto protettivo del fratello, lontano dall'intendere comeil convento rappresenti una nuova prigione per la sorella. «L'ansia di protezionedi Cosimo mi sottraeva alle mie responsabilità, $\mathrm{mi}$ riportava indietro, mi rendeva impotente, spettatrice passiva della mia vita stessa», spiega la protagonista ricordandocome la libertà l'avesse appena assaporata. Nonostante il fratello rappresenti la ragione patriarcale, è importante sottolineare come la Cutrufelli lo delinei come un personaggio comunque estremamente positivo, soprattutto, senza mai giudicarlo. Anzi, essendo il punto di vista della scrittrice interpretato dalle parole della narratriceprotagonista potremmo dire che quello di Cosimo è un personaggio amato e, a suo modo, vittima del suo tempo. Egli reagisce con stupore di fronte la forza di volontà della sorella «libera, finalmente, di compiere una scelta» e che gli ripete «io resto, resto qui con te». Cosimo fatica ad accettare questa scelta, ed è l'entrata in scena di un'altra donna, Antonia D'Acquisto, compagna o meglio "druda" di Spaziante, ad essere incisiva per l'integrazione di Margherita nella banda. «Davvero io non mi aspettavo di incontrare una donna in quel luogo e in quel momento - scrive - Guardando quel 
volto giovane e sereno sentivo allentarsi la tensione e tornare la stanchezza. Ma ora potevo abbandonarmi, le sue piccole mani mi sorreggevano con maggiore esperienza e sicurezza delle mani forti di Cosimo». Solo un'altra donna può permettere che la volontà della protagonista prenda forma, che si compia la sua "trasformazione" in brigantessa.

Sono donne queste, Margherita su tutte, che agiscono con una vera e propria coscienza femminista, essendo alla ricerca di una ricostruzione della propria identità. Una identità che non sia più ritagliata su quella maschile ma inseguita sia personalmente che con una coscienza di genere. (cfr RODRIGUEZ 2004: 135-139). La Briganta simboleggia tutte le donne che si prefiggono l'obbiettivo di inalzarsi a soggetto storico, e lo fa sia individualmente sia insieme alle sue compagne. Si converte in soggetto liberandosi da chi l'aveva limitata ad oggetto, il marito, e financo rifiutando la protezione del fratello. Partecipa alla storia andando inoltre controcorrente e sfidando il destino già scritto di un'Italia unità. Lei che si era «infervorata alle letture patriottiche, ai nobili ideali di redenzione e di unità patria», sceglie la macchia, il brigantaggio, in un certo senso la reazione. La banda di Spaziante amplia continuamente il suo organico di contadini ed ex soldati scontenti. Del capobrigante la protagonista ritrae le qualità di leader taciturno ma trascinatore di cui Cosimo, il fratello, è fidato consigliere. La rinascita di comitati borbonici fa affiorare nella banda la fievole e vana speranza di riuscire a scacciare i piemontesi dall'ex Regno della due Sicilie. Margherita si sente confortata dalla esperienza delle sua mentori e dalla forte presenza femminile nel gruppo. A procurarle i vestiti da uomo, da brigantessa è la componente più agguerrita della banda di Spaziante, Maria Orsola Cardona, detta la Bizzarra. «Si aggirava per la masseria vestita da uomo, con due revolver in cintura - la descrive la protagonista - e prendeva parte a tutte le sue scorrerie». Nel dare le vesti del "lavoro" a Margherita la assicura: «è l'abito giusto per questa vita maledetta». Il rito della vestizione viene raccontato dettagliatamente, simboleggia un cambio di status. Cresciuta in una famiglia bene, Margherita non mai aveva vissuto il vestirsi come una esperienza personale ma era stata sempre coadiuvata dalla servitù. «Ogni indumento richiedeva un gesto lunghissimo narra - non ero abituata a vestirmi senza aiuto di sorta e senza uno specchio». In questo travestimento, il mascheramento della sessualità ricopre un elemento importante: «Il seno si perdeva nella camicia bianca, larghissima, e scompariva del tutto sotto il giubbetto colorato - racconta - Poi rifeci la treccia e la nascosi sotto un berretto a cono ornato di nastri». Margherita è cosciente di non star «indossando un abito ma una vita». Tutto questo avviene sotto lo sguardo della "Bizzarra" e di Antonia che compiaciute e divertite la scrutano senza aiutarla. Sono contente che diventi "una di loro", e che sia libera in questa volontà. La neo Briganta paragona questa scena al giorno dellesue nozze quando «sguardi attenti ma preoccupati, e però con mani premurose che infilavano, allacciavano, stringevano» per acconciarli il vestito da sposa. «Tuttavia, agitata e maldestra nell'indossarlo - segue - l'avevo ugualmente lacerato: uno strappo lungo, irrimediabile». L'agitazione, l'incapacità è inadeguatezza al ruolo, insofferenza al destino: il vestito da sposa è «una maschera che mi aiutava ad ingannare la sorte, nient'altro che un gioco rassicurante». «La camicia è di vostro fratello» la informa Antonia. Margherita è felice di non potersi specchiare, come se il vedersi certificasse il tradimento che sta compiendo verso il genere femminile o forse l'espropriazione di una identità altrui: «mi sembrava di avergli rubato, insieme alla camicia, una parte della sua anima e della sua forza d'uomo giovane» dice infatti del fratello con quel senso di colpa, accentuato dal vincolo famigliare, di chi si vede uscire dal ruolo per svolgere un compito "maschile".

La Briganta della Cutrufelli è anche una donna socialmente consapevole che si scontra con le ingiustizie perpetrate dal nuovo regno d'Italia: la revoca del diritto di semina e di pascolo ad esempio. Un passo indietro «sembrava di essere tornati al 1848 denuncia - quando gli abitanti erano stati costretti ad occupare il fondo per rivendicare il diritto agli usi civici. In cento erano stati arrestati dai gendarmi del Borbone, ma Ferdinando II aveva concesso la grazia poiché li aveva ritenuti "più miseri che rei". E la popolazione di quelle montagne ancora ricordava l'atto di clemenza del sovrano e la rabbia impotente dei galantuomini». Fra le due monarchie Margherita non ha dubbi su chi fosse la più magnanima, senza per questo essere filo borbonica. La gente invece spera nel ritorno della casata e organizza manifestazioni contro le misure del nuovo governo, che vengono ogni qualvolta brutalmente represse dai piemontesi. $\mathrm{Ne}$ meridione ormai vige lo stato d'assedio e la maggior parte delle donne si preparava a partire e a lasciare gli uomini. «Eppure - scrive Margherita - in quel momento tutte si sentivano e anch'io lo sentivo di non essere legate a solo uomo ma ad una sorte comune». La difficoltà e la contingenza obbliga le donne a riscoprire la loro "sorellanza". Certo la nostra Briganta è la più ardita, e quando i bersaglieri sferrano il più duro attacco di rappresaglia nei confronti di un demanio occupato dai contadini, mentre il grosso della banda si mette al riparo, lei cavalca con Carmine verso questo terreno. «Montavo a cavallo alla maniera degli uomini, lieta che i calzoni me lo permettessero - narra - Da principio questo, per me nuovo, occupò tutta la mia attenzione, poi a poco a poco m'invase un altro sentimento, comunicatomi dall'avanzare guardingo dei miei compagni, dalla cautela di ogni loro movimento». «Per la prima volta avvertivo (...) un sentimento indefinibile, che non era semplice paura - segue - Era un'attesa spasmodica, intollerabile Era la voglia di far precipitare gli eventi per esserne, finalmente, al di là». In queste parole, Margherita esprime la paura e la gioia di esserci, di far parte della Storia, di affrontare gli ostacoli autonomamente e superarli anche in un clima diguerra. Guerra che è, per lei, il semplice «non capire dove va la propria vita». Quando finalmente arrivano al demanio lo scenario che gli si presenta è inquietante: $i$ bersaglieri avevano compiuto una vera e propria strage («forse dieci, forse più corpi umani gettati 
là come carogne»). Questa carneficina sommaria rappresenta l'ammonimento per chiunque si voglia mettere di traverso alla Storia.

A partire da questo momento, per la banda comincia la «vita randagia», che li porta a spostarsi da montagna a montagna, continuamente. «Senza mai riposo - dice Margherita - un giorno inseguendo e un giorno inseguiti». La protagonista e narratrice, è brigantessa e osservatrice. Per questo, non si esime dall'esprimere la fascinazione che subisce dalla Bizzara, brigantessa come lei ma con altre caratteristiche: più ruspante, quasi un modello da seguire nella sua rabbia contadina. «I capelli le si scioglievano sulla giacca da uomo, più fitti della criniera del cavallo. Sembrava un animale leggendario», la descrive. La Bizzarra non è solo una donna d'azione ma è anche «espertissima nel curare ferite e malattie e c'era sempre qualcuna che l'aspettava per essere medicato». La Cutrufelli sottolinea come le qualità fisiche e morali delle sue eroine si sposino quelle intellettuali: in questo caso nelle conoscenze erboristiche e paramedicinali. Carmine, il capobanda, ex fattore del padre di Margherita, è descritto come uomo taciturno e orgoglioso. Il padre della Briganta a suo tempo gli aveva prestato 200 ducati per essere esonerato dalla leva. Così che orgoglioso può dire: «Non ho mai servito sotto le bandiere (...) e riesco a sconfiggere ufficiali di carriera». L'ex contadino oraè il capobrigante; anche in questo si vede il tentativo si sovvertire l'ordine costituito. Carmine si è dato alla macchia dopo aver ucciso un uomo per strani motivi d'onore. Dal carcere si è liberato grazie alla rivoluzione garibaldina da cui però non ha ricevuto la riabilitazione sperata. Da Cosimo, suo amico, apprende a scrivere. La differenza di classe e educazione si notano, soprattutto agli occhi di una brigantessa così anomala. «Loro erano abituate a servire, io ad essere servita - dice delle sue compagne - Un'abitudine che, per la prima volta nella mia vita, avvertivo come un impaccio: di fronte a loro ero disarmata, una bambina che deve ancora imparare tutto, le cose più semplici della vita». «Senza l'aiuto della Bizzarra e di Antonia non avrei mai retto il confronto con le altre donne - confessa - Una volta accettata, con gli uomini dividevo rischi e pericoli, e questo era tutto». Il nemico è uno spauracchio, come nel Deserto dei Tartari: «non conobbi mai da vicino il nemico che mi ero scelta. Mai prima della mia cattura. - dice - Ogni tanto ci capitava di vedere, da lontano, le truppe italiane mentre arrancavano sotto il sole, le spalle gravate di quello zaino». Uno spauracchio rappresentato dal temibile capitano Crema, che rassomiglia ad uno dei tanti uomini mandati da Cavour a "debellare" il brigantaggio. Quasi un fantasma che respira «nel buio, alle nostre spalle».

All'interno della banda l'unico privilegio vantato dalle donne è essere dispensate dai turni di guardia «poiché si partecipava a tutte le altre attività della banda, nessuna esclusa - racconta Margherita - Anche noi uscivamo a turno in perlustrazione o per compiere requisizioni. E piuttosto che l'inattività forzata all'accampamento, io preferivo i rischi di queste scorrerie». L'attesa è proprio tutto ciò che la protagonista non sopporta, la porterebbe a pensare alla penosa e mortificante inattività della vita matrimoniale. Quindi si passa all'azione: la banda decide di assediare la base dei bersaglieri, i primi fuochi sono seguiti da un lungo silenzio. Al suono della tromba dei cavalleggeri Margherita reagisce sparando all'impazzando e gridando «con una voce - racconta - che non era la mia. Qualcuno mi si gettò addosso e mi trattenne a forza perfarmi smettere: era il corpo di Carmine che lottava con il mio - continua narrando - erano le sue braccia che mi tenevano, mi costringevano a tornare alla realtà, mi forzavano con il suo petto, stringevano i miei fianchi contro i suoi». L'abbraccio del capobrigante che cerca di farla rinsavire, la riporta sulla terra e le trasmette sensazioni ormai dimenticate. «Scordai il fuoco, gli uomini intrappolati, tutto l'orrore della situazione - spiega - Erano le mani di Carmine, che non m'avevanomai sfiorato prima d'allora, a toccarmi e di nuovo a stringermi in una morsa che aveva dimenticato la sua ragione d'essere - spiega - Come se fra di noi fosse stata abbattuta anche l'ultima barriera». In questo episodio avvengono due liberazioni:il battesimo del fuoco "militare" che porta Margherita ad "esplodere" il suo fucile furiosamente; e l'abbraccio del suo capobrigante che riconduce la Briganta alla terrenalibertà delle pulsioni erotiche congelate dal matrimonio. Come vestita da brigantessa di fronte allo specchio, Margherita non può riconoscersi nel suo grido di guerra.

Siamo in piena estate del 1861, impazza la guerriglia "cafona" e «la loro speranza violenta e triste». A Roma si forma un governo provvisorio di re Francesco I di Borbone, lì rifugiatosi dopo la resa di Gaeta. La notizia eccita gli animi nei briganti e Cosimo, il più istruito, viene mandato come emissario. L'allontanamento del fratello viene vissuto drammaticamente da Margherita. «Dunque, Cosimo partiva. E io restavo sola - scrive - Ma chi era l'uomo che lo allontanava da me, chi era quel Carmine Spaziante che si proclamava generale di Francesco e capo di noi tutti?».

«Un contadino che in altri tempi sarebbe stato davanti a me, davanti a Cosimo con il berretto in mano e gli occhi rispettosi», si risponde. «Ed ora (...) era Carmine Spaziante a trattare con i signori, a dettare le condizioni delle trattative, a dare gli ordini continua Margherita - A volte mi pareva un'ingiustizia, una malvagità che si fossero così capovolte le loro posizioni e le loro sorti. Al solo pensarci mi sentivo rivoltare il cuore». Margherita non accetta questo ribaltamento dei ruoli, ma è solo uno dei cambiamenti a cui non si è preparati. Eppure, anche lei di questa rivoluzione totale di valori, è complice, partecipe. Nel drammatico commiato Cosimo le rimprovera ancora una volta l'omicidio del marito: «se ti aveva offeso, non eri tu che dovevi ucciderlo dice alla sorella - E non eri tu che dovevi pagare. Perché mi hai fatto questo torto?». Aldilà del forte amore per la sorella, in Cosimo c'è l'atteggiamento paternalistica e patriarcale di chi vive nel mondo in cui gli uomini compiono i crimini, vendicano 
le "loro donne", e pagano anche loro colpe. Come nel rincontro, così nel commiato, l'incomprensione marca la relazione fra due fratelli che si vogliono bene: un uomo ancora al passato, una donna che cerca disperatamente di cambiare il presente.

Nel frattempo ricomincia la "reconquista" brigantesca: il primo paese a cadere è Torrearsa nel luglio 1861. Tuttavia, Margherita si accorge ben presto della futilità delle loro azioni: entrare nei paesi e saccheggiarli, vedendo i suoi compagni accanirsi alla ricerca di oggetti preziosi e beni di qualsiasi specie, trascina la protagonista a sentirsi intrappolata fra due sentimenti contrastanti. Da un lato «una specie di esultanza per quelle case violate e sconvolte, finalmente aperte a qualsiasi sguardo e a qualsiasi passo»; dall'altro uno «sbigottimento attonito, di malessere fisico, un urto in mezzo al petto» per quella violenza gratuita, per quella caccia cieca. Un senso di estraneità che le si ripresenta nei momenti di maggior cameratismo dei briganti. «Molte, da vecchie, hanno per professione l'essere streghe», incarna il tono delle conversazioni che le tocca ascoltare. Margherita si rende conto della buona percentuale di machismo presente nel gruppo, ma è probabile che prima di "trasformarsi" in brigantessa non sarebbe stata riuscita a riconoscerlo così chiaramente. Lo avrebbe solo sopportato. Il cameratismo macho dei briganti è esemplificato nella notte in cui Spaziante decide di portare i suoi compagni al lupanare di Fiore di Messina, una esperta "maitresse". Margherita si unisce alla "spedizione" fra la sorpresa generale e con il permesso dell'intrigato capobanda. «Davvero non so quale impulso o quale curiosità - dice - mi spingesse quella sera verso un'ambigua avventura». Lo spettacolo che si presenta agli occhi di Margherita è quello di «un ammasso indistinto di carni e sete» su cui si fiondano i virili compagni. Inizialmente, la protagonista rimane impietrita dalla situazione per poi cominciare a sentirsi trasportata «da quel palpitare nudo e scoperto, da quei seni tremanti», da cui vien attratta: «avrei voluto afferrare con le mie mani quella paura vivida e bianca - confessa - che mi affascinava e in quell' affanno terrorizzato affondare con forza le dita e la faccia e tutte me stessa». La presunta pulsione lesbica della Briganta rappresenta l'esigenza di una libertà sessuale frustrata per troppi anni, la necessità di disporre finalmente del proprio corpo. L'entrata della carismatica Fiore e la visione di alcune donne avvinghiate fanno andare nel panico la protagonista, sorpresa da quella sensazione di irrefrenabile attrazione. Margherita scappa dal lupanare: adesso non è più l'immagine del capobrigante a perseguitarla ma «il sorriso fiducioso di Antonia», adesso incinta. «Non erano le mani di Carmine a farmi rabbrividire insiste - ma la catenella d'oro che serpeggiava fra i seni di Antonia stretti nel corpetto». Solo la decisione di ferirsi al braccio con un coltello la aiuta a allentare la tensione. Nei paesi della riconquista, le donne dei paesi accolgono Carmine come un messia ma in Margherita c'e la consapevolezza che «ogni vittoriaera anche un passo avanti verso la fine di un sogno. Verso il risveglio».
«Oggi occupiamo un paese e domani è rioccupato - lamenta Spaziante - Non combineremo mai niente».

Così la vicenda di Margherita va verso la sua conclusione. La frustrazione la porta a dare fuoco a un cumulo di frasche. L'incendio attira l'attenzione dei bersaglieri che nell'inseguimento sparano e colpiscono, ferendola, la protagonista. La sua fuga, ora solitaria, la porta nel capanno di Antonia che la cura e la medica con premura. Nelle sue mani Margherita non avverte più «né il peso soffocante dell'afa né il dolore».

La festa per la riconquista del capoluogo della regione si trasforma in un epitaffio dell'esperienza brigantesca e delle sue illusioni. L'entrata trionfale in città di Carmine e della sua banda viene saluta da mortaretti e campane ma ad aspettarlo ci sono i potenti di sempre: gli aristocratici, i facoltosi, i preti. L'analisi di Margherita coglie perfettamente la gattopardiana realtà. «Isolato dai suoi, circondato dai notabiliin gran pompa, mi sembrò quasi un ostaggio, una preda, una vittima destinataal sacrificio - scrive - Era il momento del massimo trionfo, ma in quel momento io vidi di nuovo in lui il contadino e non più il generale, non più il capopopolo». In questa celebrazione non può mancare la messa. Alle donne con vestiti maschili viene proibito l'ingresso in chiesa. «Io avevo messo sopra i calzoni un'ampia gonna e questomi permetteva di entrare» narra Margherita ora consapevole di come la Chiesa sia fautrice del potere patriarcale. Infatti «il vecchio ordine tornava a rimettere a posto, secondo norme secolari e immutabili, le carte scompigliate da mani insolenti» scrive rattristata la protagonista. Il capobrigante contadino non arringa neanche la folla,che delusa si disperde. La comitiva si trasferisce in un palazzo nobiliare, la cena di "gala" per loro preparata si trasforma in festival dell'ipocrisia. Carmine rifiuta i cibi raffinati per pane cacio e uova sode, le donne sono di nuovo segregate e mangianoin cucina con la servitù. La Bizzarra confessa a Margherita: «ci chiamano briganti e briganti dobbiamo restare - dice - Sui monti, non nei palazzi, alla tavola dei signori».La nostra Briganta comunque si svaga flirtando con Antonia fra il divertimento delle serve e cameriere, negli ultimi attimi di allegro cameratismo fra brigantesse. Sono i giorni dell'abbondanza, delle grandi abbuffate e della vita da signori: «nel palazzodel conte l'argenteria e le porcellana - avverte Margherita - erano nel frattempo sparite come per l'incanto». Stando in mezzo alla servitù, la Briganta avvisa con maggiore chiarezza gli umori che lievitano e comprende come il vento stia cambiando. Informatori avvertono che i soldati italiani si stanno avvicinando al capoluogo. Il conteche da ospitalità alla banda decide abbandona il palazzo e consiglia a Carmine di darsidi nuovo alla macchia ma Spaziante, da vero generale, esita con senso di costernata rassegnazione. Se ne sta rintanato nel palazzo mentre fra gli uomini comincia «ad allentarsi la disciplina». Vanno via tutti, anche le serve. Rimane solo una vecchia che a Margherita ricorda Filomena, la serva della sua vita precedente al brigantaggio. In 
questo modo, Maria Rosa Cutrufelli, chiude brillantemente la sua struttura circolare

Gli ultimi momenti con Carmine riflettono questo ristabilire la gerarchie. Teneramente, il capobrigante chiede a Margherita di spiegarle tutte, ad una a una le rappresentazioni mitologiche degli affreschi e dei quadri che abbelliscono i saloni. Margherita si rivede in «una Sibilla bellissima con le chiome ritorte in un vortice, la veste di velo e il cuore strappato da una pallottola che aveva squarciato la tela del quadro» e riflette sul suo destino: «se un rimpianto provavo, era per tutto quello che il destino non mi aveva concesso: la tranquillità dell'animo, prima di ogni cosa. E la capacità d'oblio».

La fine dell' avventura èvicina, Antonia ha le doglie: Margherita la deve accompagnare a partorire da una lavatrice. Ma quando escono dal palazzo si ritrovano ormai circondate dai soldati italiani che aprono il fuoco da lontano e colpiscono mortalmente Antonia in pieno petto. Margherita vede un soldato levargli il fucile contro, cosi in quel momento apre il corpetto e mostra "con gesto sicuro" il seno. Grazia all' esibizionedella sua femminilità, il fucile torna ad abbassarsi. Grazie alla sua femminilità, Margherita rivendica e ottiene il diritto alla vita. La spiegazione di questa scelta è nella sua incapacità di sparare, «di reagire con tutta quella violenza che pure mi riempivail cuore. Mi era impossibile - spiega - dare la morte, ma nemmeno potevo accettarla». Nello stesso giorno in cui era scappata alla morte verrà a sapere molto tempo, e con grande dolore, che era morto il fratello, scoperto durante la sua missione romana e giustiziato dai "patrioti". Di questa morte "virile" di Cosimo, Margherita si dice gelosa e «anche colpevole». Ma in fondo, per salvarsi, la protagonista ha approfittato per una volta di quello stesso sistema patriarcale a cui si era liberata; si è avvantaggiata del prepotente privilegio del "non si spara alle donne". La Briganta finisce la sua corsa nella primavera del 1963. Dopo la cattura deve subire le violenze e le umiliazioni tipiche di una donna: «Mi frugavano con dita violente e nemiche, dicendo di volersi accertare che non nascondessi niente» racconta. «Non posso credere che fosse solo per umiliarmi - segue - Ma se lo scopo era quello di spezzare, attraverso l'umiliazione del corpo, qualsiasi resistenza (...) ci riuscirono. Alla fine provai vergogna di me e del mio corpo».

È il trattamento che la donna riceve all'interno di ogni conflitto, solo per essere donna. Margherita viene eccezionalmente condannata a morte, una pena poi commuta in ergastolo proprio per quel perdonismo fondamento del sistema patriarcale. Ma è il processo l'esperienza più dolorosa: «mi fu risparmiato soltanto la sofferenza di vedere $\mathrm{i}$ miei parenti», che non si presentano e la risparmiano della fatica di vederseli davanti. Per il giorno della sua deposizione le procurano abiti femminili, affinché sia ben chiaro che è in quanto donna che viene giudicata. In chiesa come in tribunale i ruoli vanno rispettati.
L'accusa la ritrae con i topici dell'antropologia razzista e misogina: «dall'occhio traspare la superbia del delitto, il disprezzo delle leggi della società - le dicono - Gli zigomi sporgenti, le occhiaie livide, le labbra violacee manifestano la sua immoralità, più che non provano le deposizioni del processo scritto e le orali dei testimoni». Il giudice, un uomo, le rivolge domande minuziose a cui lei non dai mai risposta. E le chiede, soprattutto, se non trovi ripugnante l'abitudine di indossare vestiti maschili. Margherita sa cosa c'è dietro quella domanda: «mi passò per la mente che forse Giovanna d'Arco era salita sul rogo anche per quel motivo, per aver indossato abiti maschili spiega - Così gelosi sono gli uomini delle loro prerogative, anche le più infime». Le altre brigantesse accusate sono cinque contadine analfabete che Margherita definisce «sbalordite e ossequienti. Le ascoltavo fornire spiegazioni dettagliate e umilianti di qualsiasi cosa fosse loro richiesta - spiega - la vita alla macchia, la provenienza dei gioielli trovati in loro possesso, anche quelle misere buccole che così chiaramente e legittimamente appartenevano alla vita di spose contadine». Margherita-Cutrufelli risalta la differenza dell'atteggiamento delle brigantesse contadine di fronte la leggeuomo, la legge-patriarcato. Queste donne «si dilungavano in storie di seduzionie rapimenti, di doveri coniugali e filiali per spiegare la loro partecipazione agliscontri e alle sollevazioni». «Sempre passive - continua - sempre trascinate loro malgrado nel gorgo della vita. Pur di salvarsi, ciascuna rinnegava se stessa e le altre. Le potevo capire». Le altre donne rinnegano mentre Margherita sceglie un ostinato silenzio. Tutte, nella finzione e nella realtà storica, coscienti o no, aggireranno la leggepatriarcale avendo salva la propria vita, proprio per essere donne. Considerate, quindi, "incapaci" di trasformarsi in soggetto storico, di essere padrone del proprio destino.

La legge, scritta e applicata dalla ragione patriarcale, fu così cieca quindi da salvare la vita proprio alle donne che, contro questo sistema, si erano ribellate. Mentre viene portata via dall'aula, nella confusione, a Margherita le sembra di ascoltare la confortante voce della Bizzarra che grida: «Sono una briganta, io, non donna di brigante». «E il ricordo di quelle parole mi dette forza e dignità», conclude la protagonista.

\section{RIFERMIENTI BIBLIOGRAFICI}

Cutrufelli, M. R., L'invenzione della donna: miti e tecniche di uno sfruttamento, Mazzotta, 1974. ----, Disoccupata con onore: lavoro e condizione della donna, Mazzotta,1975.

----, Donna perche piangi? : imperialismo e condizione femminile nell' Africa nera, Mazzotta, 1976. ----, Operaie senza fabbrica: inchiesta sul lavoro a domicilio Editori Riuniti, 1977. ----, Economia e politica dei sentimenti: "la produzione femminile". Editori Riuniti, 1980. ----, La Briganta, Palermo, La Luna, 1990. 
----, Giorni d'acqua corrente, Pratiche, 2002.

Di Giulio, C., Travesties of Risorgimento in Maria Rosa Cutrufelli's "La Briganta", Dickinson University Press, 2005.

Rossi, M., Re-thinking History: Women's Transgression in Maria Rosa Cutrufelli, Dickinson University Press, 1993.

Intervista a Maria Rosa Cutrufelli del 24 maggio 2005 pubblicato su Specchio di carta, Università di Palermo. 